\title{
Epidemiological analysis of the emergence and disappearance of the SARS-CoV-2 Kappa variant within a region of British Columbia, Canada
}

\author{
Cher Ghafari ${ }^{1 \star}$, Michael Benusic ${ }^{1}$, Natalie Prystajecky ${ }^{2}$, Hind Sbihi ${ }^{2}$ Kimia Kamelian², Linda Hoang ${ }^{2}$
}

\begin{abstract}
Background: The Kappa variant is designated as a severe acute respiratory syndrome coronavirus 2 (SARS-CoV-2) variant of interest (VOI). We identified 195 Kappa variant cases in a region of British Columbia, Canada—the largest published cluster in North America.

Objectives: To describe the epidemiology of the Kappa variant in relation to other circulating SARS-CoV-2 variants of concern (VOC) in the region to determine if the epidemiology of the Kappa variant supports a VOI or VOC status.

Methods: Clinical specimens testing positive for SARS-CoV-2 collected between March 10 and May 2, 2021, were screened for the detection of known circulating VOCs; approximately $50 \%$ of specimens were subsequently selected for whole genome sequencing (WGS). Epidemiological analysis was performed comparing the characteristics of Kappa cases to the main circulating variants in the region (Alpha and Gamma) and to non-VOC/VOI cases.
\end{abstract}

Results: A total of 2,079 coronavirus disease 2019 (COVID-19) cases were reported in the region during the study period, of which $54 \%$ were selected for WGS. The 1,131 sequenced cases were categorized into Kappa, Alpha, Gamma and non-VOC/VOI. While Alpha and Gamma cases were found to have a significantly higher attack rate among household contacts compared to non-VOI/VOC cases, Kappa was not.

Conclusion: Epidemiological analysis supports the designation of Kappa as a VOI and not a VOC. The Alpha and Gamma variants were found to be more transmissible, explaining their subsequent dominance in the region and the rapid disappearance of the Kappa variant. Variant surveillance strategies should focus on both detection of established VOCs and detection of potential new VOCs.
This work is licensed under a Creative Commons Attribution 4.0 International License.

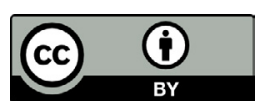

Affiliations

${ }^{1}$ Island Health, Victoria, BC

${ }^{2}$ British Columbia Centre for Disease Control, Vancouver, BC

\section{*Correspondence:}

shaherazad.ghafari@islandhealth. ca

Suggested citation: Ghafari C, Benusic M, Prystajecky N, Sbihi H, Kamelian K, Hoang L. Epidemiological analysis of the emergence and disappearance of the SARS-CoV-2 Kappa variant within a region of British Columbia, Canada. Can Commun Dis Rep 2022;48(1):22-6. https://doi.org/10.14745/ccdr.v48i01a04

Keywords: SARS-CoV-2, COVID-19, Kappa variant, variant of concern, variant of interest, variant surveillance

\section{Introduction}

The B.1.617 severe acute respiratory syndrome coronavirus 2 (SARS-CoV-2) variant was designated as the fourth variant of concern (VOC) by the World Health Organization (WHO) in May 2021 due to concerns of higher transmissibility and potential decreased effectiveness of treatment and vaccines (1). Since then, B.1.617 has been further delineated to B.1.617.2 (Delta), which remains a VOC, and B.1.617.1 (Kappa), now a variant of interest (VOI) (2). Concern remains with Kappa as a spike in cases in late May 2021 prompted control measures in Australia (3). In this article, the epidemiology of Kappa within a region of British Columbia, Canada, from March 10 to May 2, 2021 is reported in relation to other circulating variants. The objective of this analysis is to determine if the epidemiology of Kappa supports this downgrading to a VOI status, or if Kappa is epidemiologically similar to concurrently circulating VOCs. 


\section{Methods}

Between March 10 and May 2, 2021, clinical specimens testing positive for SARS-CoV-2 were screened for VOC detection by a quantitative polymerase chain reaction assay targeting the N501Y and E484K mutations in the spike gene, allowing for detection of Alpha, Beta and Gamma variants (4). If positive for N501Y, samples were presumptive positive for Alpha; if positive for N501Y and E484K, samples were presumptive positive for Beta and Gamma; if negative for N501Y and E484K, samples were not a VOC; and if negative for N501Y and positive for E484K, samples were not a VOC. Approximately $30 \%$ of VOC-positive and $20 \%$ of VOC-negative specimens were selected for whole genome sequencing (WGS).

Once Kappa was detected in the region, sequencing of select additional VOC-negative specimens was carried out based on geography and contact tracing interviews $(n=162)$. Specimens were sequenced using a 1,200 bp amplicon-based sequencing approach (5) on an Illumina MiSeq or NextSeq. The SARS-CoV-2 consensus sequences were generated using a modified Nextflow pipeline for the ARTIC network's field bioinformatics tools (6). Lineages were assigned using Pangolin (version 2.4.2, pangoLEARN (7)) and sequencing quality control (QC) metrics were assessed using nCoV-tools (version 1.5.1). Specimens with more than $85 \%$ genome coverage and no QC flags (i.e. excess ambiguity) were used in subsequent analyses. Phylogenetic analysis occurred using the Nextstrain project, an open-source platform for analyzing and visualizing genomic data. Augur version 10.2.0 and Auspice version 2.21.0 were used for bioinformatic analysis and visualization of data, respectively. Consensus sequences have been deposited to GISAID (Global Initiative on Sharing Avian Influenza Data).

Kappa was compared with non-VOCs or VOls according to WHO criteria (8), and to the main circulating VOCs in the region, Alpha and Gamma. Epidemiological indicators included pertained to demographics and criteria used to establish VOCs: transmissibility; virulence; and vaccine effectiveness. Transmissibility assessment was limited to household contacts to control for variable intensity and duration of contact that occurs in the community. Virulence was assessed by hospitalization within 14 days of specimen collection and death attributed to coronavirus disease 2019 (COVID-19) by June 1, 2021. Cases were categorized into vaccine status of either no recorded dose, partially vaccinated (at least 14 days after $1^{\text {st }}$ dose) and fully vaccinated (at least 14 days after $2^{\text {nd }}$ dose). Statistical analysis was performed comparing Kappa to Alpha and Gamma cases, Kappa to non-VOC/NOI cases, and Alpha and Gamma to non-VOC/VOI cases. Chi-square or Kruskal Wallis tests were performed using STATA (Release 16; StataCorp LLC), with statistical significance set at alpha $=0.05$.

\section{Results}

Between March 10, 2021, and May 2, 2021, 2,079 COVID-19 cases were reported in the Island Health region; $54 \%$ of specimens were selected for WGS. The proportion of specimens sent for WGS remained relatively stable throughout the study period. An epidemic curve of 1,131 sequenced cases categorized into Kappa, Alpha, Gamma and non-VOC/VOI is shown in Figure 1. The first Kappa specimen was collected on 10 March 2021 and initially detected in approximately half of sequenced cases in the Island Health region. While the number of Kappa cases per week increased and peaked at over 50 per week in the first two weeks of April 2021, the relative proportion of Kappa cases decreased due to the large relative increase of Alpha and Gamma cases. As of 14 July 2021, the last Kappa case was reported on May 2, 2021. Nineteen Delta and four Beta cases were detected and excluded from this analysis as they were relatively rare $\mathrm{VOC} / \mathrm{VOI}$ in the region during this period.

Figure 1: Number (A) and percentage (B) of COVID-19 cases sequenced specimens ${ }^{\text {a }}$ confirmed within the Island Health region of British Columbia, Canada, March 10-May 2, 2021

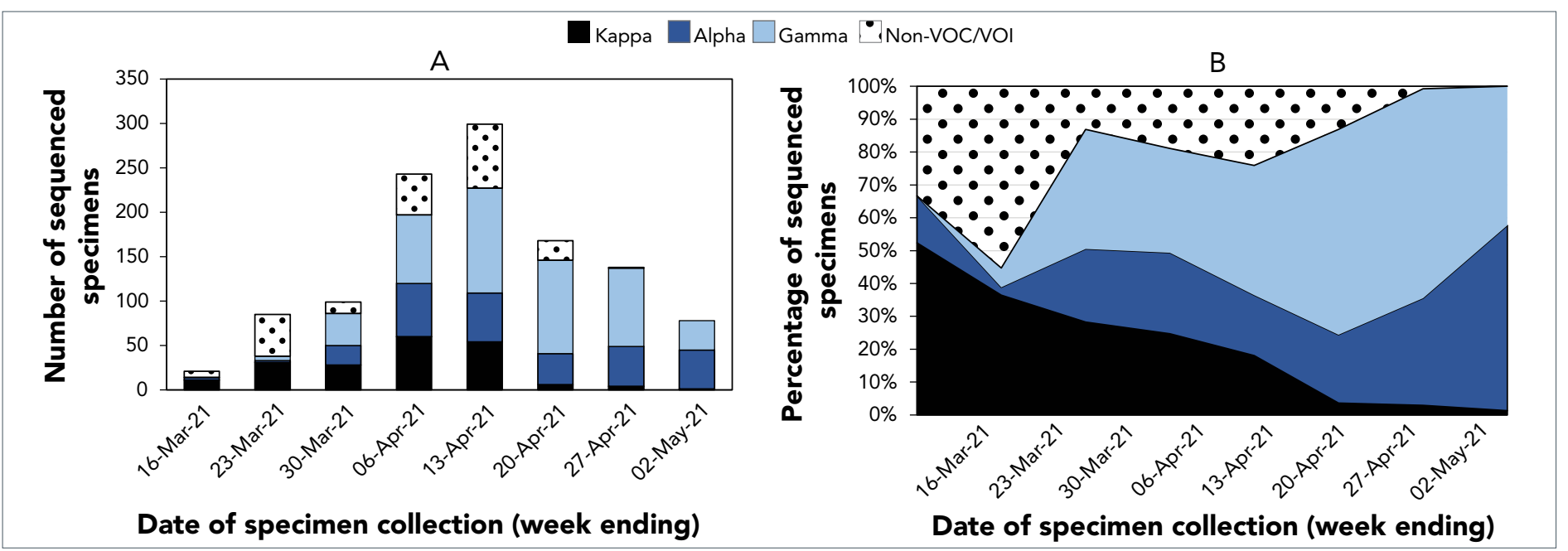

Abbreviations: COVID-19, coronavirus disease 2019; VOC, variant of concern; VOI, variant of interest

as Kappa, Gamma or non-variant of concern/variant of interest 
Table 1 compares the characteristics of Kappa, Alpha and Gamma and non-VOC/VOI cases. Age distribution was similar between Kappa and non-VOC/VOI cases, but significantly different compared with Alpha and Gamma cases $(p<0.01)$. Just over half of Kappa cases were female (52.8\%), while the majority of Alpha and Gamma and non-VOC/VOI cases were male $(53.9 \%$ and $58.2 \%$, respectively). The suspected source was similar for the three variant categories, with approximately three quarters of the cases $(73.0 \%-77.3 \%)$ linked to a confirmed case or cluster and the rest unknown. One Kappa case was linked to international travel and was not epidemiologically responsible for the primary introduction of Kappa into the region. No significant difference was detected in the attack rate among household contacts between Kappa and Alpha and Gamma (33.7\% vs. $37.7 \%$ ) or non-VOC/VOI (33.7\% vs. $27.7 \%)$. However, Alpha and Gamma had a statistically higher attack rate compared with non-VOC/VOI (37.7\% vs. $27.7 \%, p=0.01)$. Similar proportions of symptomatic cases were seen across the categories. Hospitalization rates were not significantly lower for Kappa cases compared with Alpha and Gamma cases $(1.5 \%$ vs. $4.5 \%, p=0.06)$. Case fatality rates were low $(0.5 \%-0.6 \%)$ and statistically similar across all groups. The majority of cases were unvaccinated (95.7\%-97.6\%) and no statistical differences were seen in vaccine breakthrough cases.

\section{Table 1: Comparison of characteristics of Kappa, Alpha and Gamma and non-variant of concern/variant of interest cases within the Island Health region of British Columbia, Canada, March 10-May 2, 2021}

\begin{tabular}{|c|c|c|c|c|c|c|c|c|c|}
\hline \multirow{3}{*}{ Characteristics of cases } & \multicolumn{6}{|c|}{ SARS-CoV-2 variant } & \multicolumn{3}{|c|}{$p$-value } \\
\hline & \multicolumn{2}{|c|}{ Kappa } & \multicolumn{2}{|c|}{ Alpha and Gamma } & \multicolumn{2}{|c|}{ Non-VOC/VOI } & \multirow{2}{*}{$\begin{array}{c}\text { Kappa } \\
\text { vs. } \\
\text { Alpha } \\
\text { and } \\
\text { Gamma }\end{array}$} & \multirow{2}{*}{$\begin{array}{c}\text { Kappa } \\
\text { vs. non- } \\
\text { VOC/ } \\
\text { VOI }\end{array}$} & \multirow{2}{*}{$\begin{array}{l}\text { Alpha } \\
\text { and } \\
\text { Gamma } \\
\text { vs. non- } \\
\text { VOC/ } \\
\text { VOI }\end{array}$} \\
\hline & $\mathbf{n}$ & $\%$ & $\mathbf{n}$ & $\%$ & n & $\%$ & & & \\
\hline \multicolumn{10}{|l|}{ Demographics } \\
\hline Number of cases (total=1,131) & 195 & 17.2 & 728 & 64.4 & 208 & 18.4 & $\mathrm{~N} / \mathrm{A}$ & $\mathrm{N} / \mathrm{A}$ & $\mathrm{N} / \mathrm{A}$ \\
\hline Median age in years (IQR) & 34 & $21-54$ & 33 & $23-50$ & 36 & $25-56$ & 0.63 & 0.46 & 0.10 \\
\hline \multicolumn{10}{|l|}{ Age group (years) } \\
\hline $0-17$ & 31 & 15.9 & 110 & 15.1 & 29 & 13.9 & \multirow{5}{*}{$<0.01$} & \multirow{5}{*}{0.96} & \multirow{5}{*}{$<0.01$} \\
\hline $18-44$ & 88 & 45.1 & 382 & 52.5 & 99 & 47.6 & & & \\
\hline $45-64$ & 46 & 23.6 & 178 & 24.5 & 51 & 24.5 & & & \\
\hline $65-74$ & 16 & 8.2 & 46 & 6.3 & 16 & 7.7 & & & \\
\hline $75+$ & 14 & 7.2 & 12 & 1.7 & 13 & 6.3 & & & \\
\hline \multicolumn{10}{|l|}{ Sex } \\
\hline Female & 103 & 52.8 & 336 & 46.2 & 87 & 41.8 & \multirow{2}{*}{0.10} & \multirow{2}{*}{0.03} & \multirow{2}{*}{0.27} \\
\hline Male & 92 & 47.2 & 392 & 53.9 & 121 & 58.2 & & & \\
\hline \multicolumn{10}{|l|}{ Suspected source } \\
\hline International travel & 1 & 0.6 & 1 & 0.2 & 0 & 0.0 & \multirow{3}{*}{0.34} & \multirow{3}{*}{0.58} & \multirow{3}{*}{0.39} \\
\hline Linked to confirmed case or cluster & 131 & 73.2 & 525 & 77.3 & 138 & 73.0 & & & \\
\hline Unknown & 47 & 26.3 & 153 & 22.5 & 51 & 27.0 & & & \\
\hline \multicolumn{10}{|l|}{ Transmissibility } \\
\hline Attack rate among household contacts & $67 / 199$ & 33.7 & $217 / 576$ & 37.7 & $52 / 188$ & 27.7 & 0.20 & 0.31 & 0.01 \\
\hline \multicolumn{10}{|l|}{ Virulence } \\
\hline Symptomatic & 171 & 87.7 & 629 & 86.4 & 183 & 88.0 & 0.64 & 0.83 & 0.45 \\
\hline $\begin{array}{l}\text { Hospitalized within } 14 \text { days of } \\
\text { specimen collection }\end{array}$ & 3 & 1.5 & 33 & 4.5 & 8 & 3.9 & 0.06 & 0.16 & 0.67 \\
\hline Deaths attributed to COVID-19 & 1 & 0.5 & 5 & 0.6 & 1 & 0.5 & 0.79 & 0.96 & 0.64 \\
\hline \multicolumn{10}{|l|}{ Vaccine effectiveness } \\
\hline No recorded dose & 188 & 96.4 & 697 & 95.7 & 203 & 97.6 & \multirow{3}{*}{0.83} & \multirow{3}{*}{0.48} & \multirow{3}{*}{0.45} \\
\hline Partially vaccinated & 7 & 3.6 & 30 & 4.1 & 5 & 2.4 & & & \\
\hline Fully vaccinated & 0 & 0 & 1 & 0.1 & 0 & 0 & & & \\
\hline
\end{tabular}

Abbreviations: COVID-19, coronavirus disease 2019; IQR, interquartile range; N/A, not applicable; SARS-CoV-2, severe acute respiratory syndrome coronavirus 2; VOC, variant of concern;

VOI, variant of interest

a 84 cases were excluded where no exposure category was recorded, representing $7 \%-9 \%$ of each of the variant categories 
Figure 2 provides a phylogenetic tree of Kappa cases in this study. Amongst the 195 cases, seven distinct genomic clusters were identified. The genomic clusters mirrored geographic distribution of cases and were consistent with transmission patterns identified through contact tracing interviews. The genomic clusters did not include any cases from other regions of British Columbia. None of the cases in the seven genomics clusters had documented travel histories.

\section{Discussion}

While responsible for more than one in six cases over the study period, approximately one third of cases for the first three weeks of the study, and seven distinct genomic and epidemiological clusters, Kappa has disappeared from the region with the last case detected on May 2, 2021. During the same period, Alpha and Gamma became the dominant strains in the region. The finding that Alpha and Gamma had a statistically higher attack rate among household contacts than non-VOC/VOI suggests that collectively these variants are more transmissible, supported by their designation as VOCs. This higher transmissibility may explain why Alpha and Gamma seemed to have outcompeted Kappa, which had a household attack rate statistically similar to non-VOC/VOIs. A similar trend was observed in the United Kingdom where, despite an initial rapid increase in the proportion of Kappa cases, Kappa is now responsible for fewer than $0.1 \%$ of recent VOC/NOI cases (8). In India, the proportion of sequenced cases were over 40\% Kappa variant in March 2021, but has now fallen to less than $20 \%$ as Delta variant has dominated (9).

The lower case hospitalization rate for Kappa compared with Alpha and Gamma seen in this study is consistent with United Kingdom findings where $1.0 \%$ of Kappa cases have been hospitalized, compared with $2.8 \%$ of Alpha cases, and none of the over 400 cases of Kappa have died (10). While no difference in vaccine breakthrough cases were seen between Kappa and non-VOC/VOI cases in this study, a study using serum from vaccinated individuals showed 2.7 -fold reduction in geometric mean neutralization titers against the Kappa variant compared with a non-VOC/VOI strain (11).

Given that Kappa was not circulating in Canada prior to its detection in this region of British Columbia, it is assumed to have been introduced via international travel. The genomic cluster data suggest a single introduction of Kappa into the region, though none of the cases had travel histories. The collection date for the earliest sample (March 10, 2021) is after the Canadian government introduced mandatory testing and quarantine for returning travellers (February 22, 2021). It is possible that the initial introduction preceded the introduction of this program and was not captured through routine testing.

\section{Limitations}

This study is likely underpowered, which poses a challenge to determine small differences between Kappa, Alpha and Gamma, and non-VOC/NOI cases. During the study period, there was also high coverage of first dose mRNA vaccine among the most vulnerable (particularly those over 70 years old), and an overall first dose vaccine coverage that rose from $\sim 10 \%$ to $25 \%$ for adults in the region, which likely reduced the relative risk of infection and severity among vulnerable people, reducing the power of this study to provide distinctions among variant categories.

\section{Conclusion}

Conclusions regarding virulence and vaccine effectiveness are difficult to make due to hospitalizations, deaths and vaccine breakthrough cases being relatively rare events; however, the epidemiology of Kappa within the Island Health region of British

Figure 2: SARS-CoV-2 genetic diversity for Kappa cases within the Island Health region of British Columbia, Canada, March 10-May 2, 2021 ${ }^{a}$

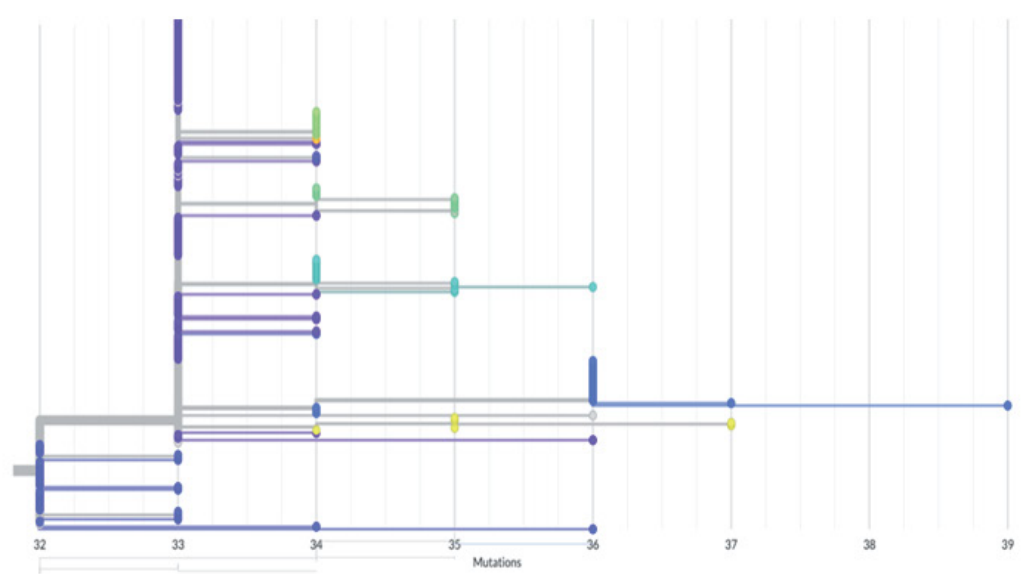

Abbreviation: SARS-CoV-2, severe acute respiratory syndrome coronavirus-2

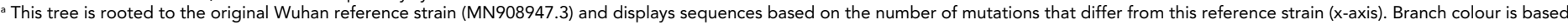
on genetic relatedness, as determined using the Cluster Picker algorithm 
Columbia, Canada, supports the assertion by WHO that Kappa does not meet the VOC criteria.

Lastly, the variant surveillance described here emphasizes the importance of performing WGS on at least a portion of cases that are categorized as non-VOC based on polymerase chain reaction of N501Y and E484K mutations. Without WGS of these samples, it is unlikely that Kappa or Delta would have been identified within the region, which guided targeted WGS based on epidemiological links to monitor spread. A similar variant surveillance strategy could be utilized with the intention of maintaining detection of variants which may not share the common mutations found in Alpha, Beta and Gamma variants.

\section{Authors' statement}

CG and $\mathrm{MB}$ - Analyzed the epidemiological data NP, HS, KK, LH - Analyzed the genomic data

All authors contributed to the conceptualization, methodology and writing of the manuscript.

The content and view expressed in this article are those of the authors and do not necessarily reflect those of the Government of Canada.

\section{Competing interests}

None.

\section{Acknowledgements}

The Island Health Case and Contact Management Surveillance team and Dr. S Allison were involved in management of the cases referred to in this study. Staff at the British Columbia Centre for Disease Control Public Health Laboratory performed variant of concern (VOC) qPCR and sequencing of severe acute respiratory syndrome coronavirus 2 (SARS-CoV-2) clinical specimens. Dr. A Jassem and Dr. J Tyson contributed to VOC screening and whole genome sequencing testing.

\section{Funding}

None.

\section{References}

1. World Health Organization. Weekly epidemiological update on COVID-19 - 11 May 2021. Geneva (CH): WHO; May 2021 (accessed 2021-07-21). https://www.who.int/publications/m/ item/weekly-epidemiological-update-on-covid-19---11may-2021
2. World Health Organization. Weekly epidemiological update on COVID-19 - 1 June 2021. Geneva (CH): WHO; June 2021 (accessed 2021-07-21). https://www.who.int/publications/m/ item/weekly-epidemiological-update-on-covid-19---1-june-2021

3. Australian Government Department of Health. Greater Melbourne declared a hotspot for Commonwealth support. Canberra, Australia, AGDH; May 2021 (accessed 2021-07-21). https://www.health.gov.au/news/greater-melbourne-declared-ahotspot-for-commonwealth-support

4. Hogan CA, Jassem AN, Sbihi H, Joffres $Y$, Tyson JR, Noftall $K$ Taylor M, Lee T, Fjell C, Wilmer A, Galbraith J, Romney MG, Henry B, Krajden M, Galanis E, Prystajecky N, Hoang LM. Rapid Increase in SARS-CoV-2 P.1 Lineage Leading to Codominance with B.1.1.7 Lineage, British Columbia, Canada, January-April 2021. Emerg Infect Dis 2021;27(11):2802-9. DOI PubMed

5. Freed NE, Vlková M, Faisal MB, Silander OK. Rapid and inexpensive whole-genome sequencing of SARS-CoV-2 using 1200 bp tiled amplicons and Oxford Nanopore Rapid Barcoding. Biol Methods Protoc. 2020;5(1):bpaa014. DOI

6. Galloway SE, Paul P, MacCannell DR, Johansson MA, Brooks JT, MacNeil A, Slayton RB, Tong S, Silk BJ, Armstrong GL, Biggerstaff M, Dugan VG. Emergence of SARS-CoV-2 B.1.1.7 Lineage - United States, December 29, 2020-January 12, 2021. MMWR Morb Mortal Wkly Rep 2021;70(3):95-9. DOI PubMed

7. Rambaut A, Holmes EC, O'Toole Á, Hill V, McCrone JT, Ruis C, du Plessis L, Pybus OG. A dynamic nomenclature proposal for SARS-CoV-2 lineages to assist genomic epidemiology. Nat Microbiol 2020;5(11):1403-7. DOI PubMed

8. World Health Organization. Tracking SARS-CoV-2 variants Geneva (CH): WHO (updated 2021-12; accessed 2021-07-21). https://www.who.int/en/activities/tracking-SARS-CoV-2-variants/

9. Singh J, Rahman SA, Ehtesham NZ, Hira S, Hasnain SE. SARS-CoV-2 variants of concern are emerging in India. Nat Med 2021;27(7):1131-3. DOI PubMed

10. Public Health England. SARS-CoV-2 variants of concern and variants under investigation in England. Technical briefing 15. London (UK): PHE; June 2021 (accessed 2021-07-21). https:// assets.publishing.service.gov.uk/government/uploads/system/ uploads/attachment_data/file/993879/Variants_of_Concern VOC_Technical_Briefing_15.pdf

11. Liu C, Ginn HM, Dejnirattisai W, Supasa P, Wang B, Tuekprakhon A, Nutalai R, Zhou D, Mentzer AJ, Zhao Y, Duyvesteyn HM, López-Camacho C, Slon-Campos J, Walter TS, Skelly D, Johnson SA, Ritter TG, Mason C, Costa Clemens SA, Gomes Naveca F, Nascimento V, Nascimento F, Fernandes da Costa C, Resende PC, Pauvolid-Correa A, Siqueira MM, Dold C, Temperton N, Dong T, Pollard AJ, Knight JC, Crook D, Lambe T, Clutterbuck E, Bibi S, Flaxman A, Bittaye M, Belij-Rammerstorfer S, Gilbert SC, Malik T, Carroll MW, Klenerman P, Barnes E, Dunachie SJ, Baillie V, Serafin N, Ditse Z, Da Silva K, Paterson NG, Williams MA, Hall DR, Madhi S, Nunes MC, Goulder P, Fry EE, Mongkolsapaya J, Ren J, Stuart DI, Screaton GR. Reduced neutralization of SARS-CoV-2 B.1.617 by vaccine and convalescent serum. Cell 2021;184(16):4220-4236.e13. DOI PubMed 